\section{Neurowissenschaftliche Begutachtung}

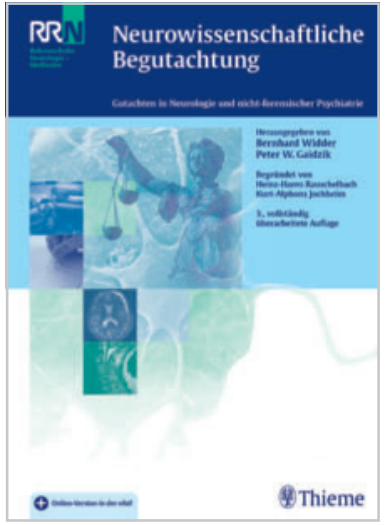

Bernhard Widder, Peter Gaidzik (Hrsg). Neurowissenschaftliche Begutachtung - Gutachten in Neurologie und nicht-forensischer Psychiatrie. Stuttgart: Thieme Verlag 2018. 3. vollständig überarbeitete Auflage, 696 Seiten, 75 Abbildungen, 169,99 Euro, ISBN 9783131407030

Alle, die in der neurologischen Begutachtung tätig sind, kennen (oder sollten zumindest kennen) das Werk, das von HeinzHarro Rauschelbach und Kurt-Alphons Jochheim unter dem Titel „Die neurologische Begutachtung“ begonnen worden ist. Nach der 4. Auflage unter diesen Herausgebern ist das Werk 2007 in die Referenzreihe Neurologie unter dem Titel „Begutachtung in der Neurologie“ übergegangen, herausgegeben von Bernhard Widder und Peter W. Gaidzik. Jetzt liegt die 3. Auflage innerhalb der Referenzreihe Neurologie vor, der Titel: „Neurowissenschaftliche Begutachtung“. Dies trägt der Tatsache Rechnung, dass die Grenzen der Begutachtung zwischen Neurologie und Psychiatrie/Psychosomatik fließend sind und es daher gerade für sozialmedizinische Gutachten sinnvoll ist, beide Fachgebiete gemeinsam abzuhandeln. Ausgenommen ist die forensische Psychiatrie, die hier keine Überschneidungen hat.

Der bewährte Aufbau in Grundlagen der Begutachtung (insbesondere aus juristischer Sicht), in Begutachtung in verschiedenen Rechts- und Versorgungsbereichen, in die Zustandsbegutachtungen und schließlich in die Zusammenhangsbegutachtung wurde beibehalten. Am Ende wird noch auf die Besonderheiten in Österreich und in der Schweiz eingegangen, außer- dem finden sich die Bewertungstabellen, z. B. für GdB und für MdE, die eine verlässliche Orientierung bieten. Inhaltlich ist diese Auflage gegenüber der vorherigen behutsam überarbeitet worden. Dabei tragen die Überarbeitungen den Veränderungen vor allem im Sozialrecht Rechnung; auch neue Begutachtungsgebiete wie z. B. die Begutachtung von Migranten muss hier genannt werden. Behutsam ist die Überarbeitung deswegen, weil gerade bei den Bewertungsmaßstäben im Sozialrecht und privaten Unfallrecht nicht immer neue Zahlen in den Raum gestellt werden dürfen, um Ungerechtigkeiten zu vermeiden. Besonders bemerkenswert ist wieder der Hinweis auf mögliche Fehler in der Begutachtung selbst und in der Beurteilung der Probanden.

Es ist bei Rezensionen immer Vorsicht geboten, von „Standardwerken“ oder „Referenzwerken“ zu sprechen. In diesem Fall muss man dies aber eindeutig tun. Dieses Buch ist in seiner langen Tradition das Standardwerk der neurowissenschaftlichen Begutachtung schlechthin. Es ist allein ausreichend und umfassend für den Hintergrund einer seriösen Begutachtung auf dem Gesamtgebiet der Neurologie und Psychiatrie (ohne Forensik). Es ist für die Bewertung von Schädigungsfolgen und Krankheiten welcher Art auch immer das Referenzwerk. Insofern darf dieses Buch wirklich in keiner Bibliothek zur Begutachtung in Neurologie und Psychiatrie fehlen.

Stefan Evers, Coppenbrügge

\section{Interkulturelle Kommunikation in der Medizin}

Anton Gillessen et al. (Hrsg). Interkulturelle Kommunikation in der Medizin. Berlin: Springer Verlag 2020. 349 Seiten, mit Abbildungen, 49,99 Euro, ISBN 9783662590126

Unstreitig ist die Interkulturelle Kommunikation in der Medizin von zunehmender Bedeutung, nicht nur auf der verbalen Ebene, sondern auch auf der metaverbalen und auf der Verständnisebene. Dies wird in den letzten Jahren immer mit der großen Zahl von Geflüchteten in unserem Land verbunden. Es ist aber schon seit 1945 ein wichti- ges Thema in der Medizin, spätestens mit den eingeladenen „Gastarbeitern“, die als interkulturelles Problem in den ersten Jahrzehnten aber nicht wahrgenommen wurden. Insofern ist es verdienstvoll, dass die Ärztekammer Westfalen-Lippe ein Curriculum entwickelt hat, in dem „Transkulturelle Medizin" vermittelt wird. In diesem Kontext ist der hier vorgelegte Sammelband entstanden, viele Autoren sind auch Referenten in dem Curriculum. Es handelt sich hierbei nicht um ein eigentliches Lehrbuch oder eine Monografie, sondern um eine Sammlung von Aufsätzen, die in den Kontext passen und von denen viele aus dem Curriculum entstanden sind.

Im ersten Abschnitt geht es um Grundlagen der Migration und um deren Auswirkungen auf die Kommunikation in der Medizin. Im zweiten Abschnitt werden Sprachbarrieren behandelt, wobei nicht nur die Sprachbarrieren gemeint sind, die z. B. Geflüchtete natürlicherweise in Deutschland haben, sondern auch Sprachbarrieren bei den Mitarbeitern im Gesundheitswesen, die in Deutschland arbeiten möchten (und dringend benötigt werden, ja sogar gar nicht mehr wegzudenken sind). Die Problematik unseres Gesundheitssystems, das auf bestimmte Bedürfnisse von Geflüchteten nicht immer adäquat eingestellt ist, wird im dritten Abschnitt behandelt. Schließlich werden zahlreiche Beispiele von Problemen in der interkulturellen Kommunikation in verschiedenen Fachbereichen eher exemplarisch dargestellt. Hauptfelder sind die Gynäkologie, Neurologie und Psychiatrie. Schließlich wird ein eigener Abschnitt am Ende den verschiedenen Aspekten der Suchterkrankungen bei Geflüchteten gewidmet. Am Ende des Buches wird ein Serviceteil angekündigt, der aber nur aus einem Stichwortverzeichnis besteht. Immer wieder werden in dem Buch Exkurse eingestreut, die sich mit ganz speziellen Aspekten beschäftigen und oft religionssystematisch orientiert sind.

Das Buch eignet sich für alle, die an interkultureller Kommunikation auf allen Ebenen interessiert sind zur Vertiefung von einzelnen Aspekten. Es ist flüssig geschrieben und editiert.

Stefan Evers, Coppenbrügge 